\title{
El impacto de las tasas retributivas para el control de vertimientos en Colombia
}

\author{
Impact of Retributive Taxes for Spills \\ Control in Colombia
}

\section{O impacto dos impostos retributivos para o controle de derramamentos na Colômbia}

\author{
Johanna Mildred Méndez-Sayago * \\ Jhon Alexánder Méndez-Sayago ** \\ Hugo Alfonso Hernández-Escolar ${ }^{* * *}$
}

DOI: https://doi.org/10.19053/01203053.v36.n64.2017.5314

Fecha de recepción: 30 de agosto de 2016

Fecha de aceptación: 25 de mayo de 2017

* Ingeniera de Producción Industrial. Magíster en Economía. Profesora de la Facultad de Estudios a Distancia, Universidad EAN, Bogotá, Colombia.jmmendez@correo.ean.edu.co iD http://orcid.org/0000-0003-33063449

** Ingeniero Civil. Doctorado en Economía del Desarrollo, FLACSO. Profesor asistente de la Facultad de Ciencias Sociales y Económicas de la Universidad del Valle. Cali, Colombia. jhon.mendez@correounivalle.edu.co (iD) http://orcid.org/0000-0003-4584-2749

${ }^{* * *}$ Economista. Magíster en Economía. Profesor de la Corporación Universitaria del Meta. Villavicencio, Colombia. hahernandeze@hotmail.com (D) http://orcid.org/0000-0003-2735-2666 


\section{Resumen}

En este artículo se discute la habilidad de las técnicas econométricas de antecedente, para evaluar el impacto de las tasas retributivas como instrumento para el control de los vertimientos en Colombia. Con este propósito se estima el efecto de las tarifas sobre la contaminación a partir de dos estrategias, que difieren en cuanto al tipo de información que se utiliza como variable de tratamiento. En la primera estimación se emplea un conjunto de datos panel, resultado de la implementación de las tasas retributivas en la jurisdicción de CORNARE durante el periodo 2011-2014. En esta primera evaluación, el precio a la contaminación es la tarifa que cobra la autoridad ambiental. En el segundo caso, la información proviene de una encuesta aplicada a empresas con vertimientos en la ciudad de Bogotá durante el año 2014. Para este caso, la variable de tratamiento es el precio percibido por los agentes contaminadores. Se encontró que la primera estrategia subestima el efecto de las tarifas, porque no captura el efecto heterogéneo del precio a la contaminación, lo que genera estimadores sesgados e inconsistentes de su impacto.

Palabras clave: tasas retributivas, control de vertimientos, endogeneidad.

Clasificación JEL: Q53, C52, C10. 
Apuntes CENES Volumen 36, Número 64

julio - diciembre 2017. Págs. 167-198

\section{Abstract}

This article discusses the ability of traditional econometric estimates of the impact of the retributive taxes as an instrument for spill control in Colombia. For this purpose the effect of fees on pollution is estimated based on two strategies that differ in the type of information that is used as a treatment variable. The first estimate uses a panel data set resulting from the implementation of the retributive taxes in CORNARE's jurisdiction during the period 2011-2014. In this first evaluation the price of the pollution is the rate that charges the environmental authority. In the second case the information comes from a survey applied to companies with spills in the city of Bogotá during the year 2014. For this case the treatment variable is the price perceived by the polluting agents. It was found that the first strategy underestimates the effect of fees because it does not capture the heterogeneous effect of price to pollution and this generates biased and inconsistent estimators of its impact.

Keywords: retributive taxes, spill control, endogenity. 


\section{Resumo}

Este artigo discute a capacidade de técnicas econométricas para avaliar a história impacto das Tasas Retributivas como uma ferramenta para controlar as descargas na Colômbia. Para este efeito, o efeito de taxas de poluição de duas estratégias que diferem no tipo de informação que é usada como uma variável de tratamento é estimado. Na primeira estimativa é utilizado um conjunto de dados de painel resultado a aplicação das Tasas Retributivas na jurisdição da CORNARE durante o período de 2011-2014. Neste primeiro poluição preço de avaliação é a taxa cobrada pela autoridade ambiental. No segundo caso, a informação vem de um levantamento das empresas com vertimientos em Bogotá durante 2014. Neste caso, a variável de tratamento é o preço recebido pelos poluentes.. Verificou-se que a primeira estratégia subestima o efeito de taxas, porque não captar o efeito heterogêneo de preço sobre a poluição e isso gera estimativas enviesados e inconsistentes de seu impacto.

Palavras-chave: taxas de descarga, controle de dumping, endogeneidade. 
Apuntes CENES Volumen 36, Número 64

julio - diciembre 2017. Págs. 167-198

\section{INTRODUCCIÓN}

Las actividades humanas productivas y no productivas contaminan el medio ambiente. Esta relación entre el hombre y el medio natural no se rige por el mercado, así que, sin la intervención del gobierno, el uso del medio ambiente como vertedero puede sobrepasar su capacidad de asimilación, produciendo efectos negativos sobre el bienestar de las comunidades, generando costos a los productores y a los consumidores, y deteriorando los ecosistemas. Dado que la contaminación afecta el bienestar y reduce la productividad, los agentes que la generan deben asumir los costos que implica eliminar o mitigar los impactos negativos que causan sus vertimientos.
Existen tres fuentes principales de contaminación del agua: las aguas negras domésticas, los e luentes industriales y la escorrentía por el uso del suelo. En la década de los noventa, en Colombia ya se había identi icado la contaminación hídrica como uno de los problemas ambientales de mayor gravedad para la sociedad colombiana (Villegas, Castiblanco, Berrouet \& Vidal, 2006). El vertimiento de aguas negras de origen urbano y rural se cuanti icó en un aporte de carga orgánica de 1.200 toneladas de DBO/día, debido a que el $95 \%$ de aguas residuales domésticas y agrícolas se vertía sin tratamiento alguno. El $85 \%$ de las aguas residuales industriales se vertía sin tratamiento adecuado, resultando en una contaminación industrial de 520 toneladas de $\mathrm{DBO} /$ día (O icina de Análisis Económico del Ministerio del Medio Ambiente, 1997). 
El tratamiento y la disposición inadecuados de las aguas residuales generaron una creciente problemática de contaminación ambiental y sanitaria, principalmente en las fuentes abastecedoras de agua, limitando así la disponibilidad del recurso hídrico y restringiendo su uso en el país. Para obtener la calidad ambiental deseada por la sociedad al mínimo costo económico posible, la Ley 99 de 1993 y el Decreto Reglamentario 901 de abril de 1997 profundizan el uso del instrumento económico denominado tasa retributiva.

El estudio nacional del agua del IDEAM (2014) registra que en el 2012 se estimó que la materia orgánica biodegradable (DBO) vertida a los cuerpos de agua es de 756.945 toneladas/año, mientras que la materia orgánica no biodegradable, es decir, sustancias químicas, es de 918.670 toneladas/año. También 205 toneladas/año de mercurio son vertidas al suelo y al agua de los ríos en el ámbito nacional. En cuanto a materia orgánic a, la cifra del 2012 supera las 627.800 toneladas/año de DBO en la década de los 90. Este crecimiento de la contaminación por materia orgánica puede ser producto del aumento de la contaminación de origen doméstico generado por el incremento de la población, también puede ser causado por un incremento de la producción o un indicador de la ineficacia del programa de tasas retributivas.

Atendiendo al incremento registrado en los vertimientos cabe preguntarse si las tasas retributivas son un instrumento eficaz para el control de la contaminación de los cuerpos de agua en Colombia. La respuesta a esta pregunta demanda determinar el efecto que tiene la tarifa de la tasa retributiva sobre la concentración de sustancias contaminantes en los vertimientos. El principal aporte de este artículo es desvelar y avanzar en superar las limitaciones de las evaluaciones de impacto de las tasas retributivas de antecedente.

En teoría, los instrumentos de precios a la contaminación, como las tasas retributivas, inducen a los agentes contaminadores a ejercer mayores esfuerzos de reducción, porque entre más alto sea el precio marginal, el abatimiento les resulta menos costoso que el pago por contaminar. A propósito, Rudas (2005) advierte que para que las tasas retributivas tengan el efecto deseado de reducción de la contaminación, la tarifa de la tasa se debe fijar en un nivel que efectivamente induzca al usuario a un uso más eficiente del recurso.

Es obvio que el conocimiento de la tarifa de la tasa es un elemento importante para que los agentes tomen decisiones óptimas ${ }^{1}$ de abatimiento. Méndez

\footnotetext{
${ }^{1}$ Minimizadora de costos por contaminar, lo que comprende el pago por concepto de la tasa y el costo de abatimiento.
} 
y Hernández (2012) desarrollaron un estudio exploratorio para identificar el mecanismo de acción de las tasas retributivas. Encontraron que el $94 \%$ de las empresas encuestadas no conocía las tarifas de la tasa retributiva, por lo tanto se deduce que este instrumento de la normatividad ambiental colombiana no funciona de acuerdo con la predicción teórica. Concluyeron que las decisiones de reducción de los agentes contaminadores obedecen más a su percepción de la tasa retributiva como una sanción por contaminar, que al incentivo económico de precios.

Los hallazgos de Méndez y Hernández (2012) sugieren que el impacto de la tarifa es heterogéneo entre agentes contaminadores. El efecto es nulo para las empresas que no se preocupan por conocer su valor y si induce abatimiento en caso contrario. El efecto marginal estimado de la tarifa tampoco es el efecto promedio, porque es muy probable que el efecto dependa de la concentración de la contaminación o la carga contaminante del usuario (endogeneidad). Esto puede invalidar los resultados de las evaluaciones de antecedente, que asumen que el efecto de la tasa sobre la contaminación es homogéneo, como por ejemplo Galarza (2009). Por tanto, este artículo pretende dar respuesta a la siguiente pregunta subsidiaria ila heterogeneidad en el conocimiento y percepción de las tarifas de la tasa retributiva por parte de los agentes contaminadores genera estimaciones sesgadas de su impacto?

Para responder esta última pregunta, el artículo presenta argumentación teórica y evidencia empírica que pone en duda la fiabilidad de las estimaciones econométricas de antecedente. La evidencia empírica que se expone en este documento sobre el impacto de las tarifas de las tasas retributivas en el control de vertimientos, utiliza dos conjuntos diferentes de información y técnicas distintas de estimación.

La información del primer conjunto de datos proviene de CORNARE (Corporación Autónoma Regional de las Cuencas de los Ríos Negro y Nare) en Colombia. De su página web se descargó información para cada usuario con vertimientos: el factor regional aplicado, el caudal vertido (en $\mathrm{m}^{3} / \mathrm{año}$ ) y las cargas de DBO y sólidos suspendidos totales - SST (en kg/año), desde el año 2010 al 2014. El número de usuarios es superior a doscientos en cada periodo. La estimación del impacto de las tasas retributivas se hizo utilizando técnicas para datos panel. Se trata de una regresión de la concentración de las sustancias contaminantes sobre los valores reportados por CORNARE de los factores regionales de la tasa retributiva. Esta autoridad ambiental es líder en el proceso de socialización e 
implementación de las tasas retributivas (Quintero, 2007; Ministerio del Medio Ambiente, 2002) y la información es abierta para el público.

La segunda técnica de evaluación del impacto de las tasas retributivas se lleva a cabo mediante una regresión de la reducción de la concentración de las sustancias contaminantes en la planta de tratamiento, sobre el incentivo económico de precio a la contaminación. La principal diferencia es que el regresor toma el valor de cero si el agente contaminador no conoce el valor de la tarifa de la tasa. Para esto se aplicó una encuesta con el propósito de determinar el conocimiento y la percepción de los agentes contaminadores sobre la tasa retributiva. El número de encuestados fue de 315 funcionarios del departamento de gestión ambiental de empresas con vertimientos en Bogotá, Colombia. Esta segunda aproximación permite que el efecto de la tarifa sea heterogéneo, nulo para las empresas que ignoran la tarifa $y$, por lo tanto, no toman decisiones de reducción de sus vertimientos en función del precio a la contaminación, y teóricamente positivo en caso contrario.

En la primera estimación se encontró que la tarifa de la tasa de sólidos suspendidos totales (SST) sí tiene efecto sobre la concentración de la contaminación de la misma sustancia contaminante en los efluentes de las firmas. Esto puede ser atribuido a que la tarifa de la tasa por SST alcanza un valor promedio más alto que la de DBO y existe un mayor porcentaje de firmas con tarifas de SST por encima de la tarifa mínima en comparación con las tarifas de DBO. En la segunda estrategia de estimación se encontró efecto positivo y significativo de las tarifas por DBO y SST sobre la reducción de la concentración de estas sustancias contaminantes en la planta de tratamiento.

El artículo está organizado de la siguiente forma: el primer apartado define las tasas retributivas e ilustra el efecto teórico de la tarifa de la tasa retributiva sobre la contaminación. También presenta un análisis desde la teoría económica de la evolución de la normatividad sobre las tasas retributivas. El segundo apartado explica cómo, en teoría, el efecto dual de la tarifa trae consecuencias sobre la estimación econométrica del impacto del precio a la contaminación y discute la literatura previa sobre la evaluación de las tasas retributivas. El tercer apartado presenta las estrategias empíricas para la evaluación de impacto. El apartado cuatro revela los resultados, y en último término se presentan las conclusiones.

\section{TASAS RETRIBUTIVAS}

La tasa retributiva es un pago que cobra la autoridad ambiental competente en Colombia, a quienes utilizan el re- 
curso hídrico como receptor de vertimientos puntuales directos o indirectos (Velásquez, 2002).

Rudas (2008) aclara que el instrumento tiene una doble función. Envía una señal de precio a los usuarios de los recursos naturales, incentivándolos a un uso más eficiente del que harían si pudieran acceder a ellos de manera gratuita. También se constituye en fuente de ingresos para financiar la política ambiental, mediante la captación de recursos financieros pagados por los usuarios directos de los recursos naturales

El Decreto 2667 del 2012 es la norma que contiene la reglamentación vigente sobre las tasas retributivas, y en el artículo 18 precisa la fórmula para el cálculo del monto por pagar $M P$ por concepto de la tasa retributiva:

$$
M P=\sum_{\mathrm{i}=1}^{n} T_{m i} * F r_{i} * C_{i}
$$

Donde:

$T_{m i}$ : Tarifa mínima del parámetro.

$\mathrm{Fr}_{i}$ : Factor regional del parámetro aplicado al usuario.

$C_{i}$ : Carga contaminante del parámetro.

$n$ : Parámetros sujetos de cobro. Son la demanda bioquímica de oxigeno (DBO) y sólidos suspendidos totales (SST).

El producto $T_{m i} * F r_{i}$ corresponde a la tarifa de la tasa (precio de la contaminación). Este par de factores le permiten al instrumento funcionar de acuerdo con el sistema de estándar y precios de Baumol y Oates (1971). El primer componente es una tarifa mínima que estableció el Ministerio del Medio Ambiente en la Resolución 273 de 1997 y se actualiza anualmente de acuerdo con el índice de precios al consumidor. El segundo es un factor regional que puede incrementarse semestralmente y tiene como propósito hacer más costosa la contaminación a los usuarios paulatinamente, hasta que sea posible alcanzar una meta de contaminación definida por la autoridad ambiental.

La Figura 1 ilustra un escenario probable para el efecto de la tarifa de la tasa retributiva sobre la contaminación de las empresas. El eje El eje $x$ corresponde a la variable de contaminación $e$. Se asume la existencia de dos firmas con costos marginales de reducción de la contaminación $C M R_{1}$ y $C M R_{2}$, con los costos representados en el eje $y$. Obsérvese que para la firma 2 es más costoso reducir cada unidad adicional de contaminación. Supóngase también que el nivel inicial de contaminación para ambas firmas es $e^{0}$, ya sea porque es su meta de contaminación fijada por la autoridad ambiental (una meta baja) o porque su responsabilidad ambiental la lleva a tener algún nivel de reducción de la contaminación. Ahora considere que la tarifa de la tasa es 
$t=t m * F r$. Ante el cobro de este precio a la contaminación, las firmas minimizadoras de costos reducen su contaminación hasta el punto en que el precio a la contaminación iguala el costo marginal de reducción de la contaminación. Obedeciendo a este criterio, la contaminación final será $e_{1}^{f}<e^{o}$ para la firma 1 y $e^{f}>e^{o}$. El impacto de la tarifa de la tasa para la firma 1 es una reducción de la concentración de la contaminación de magnitud $e^{f}-e^{o}$. La firma 2 no reduce la contaminación, sus costos marginales de reducción son tan altos que para esta magnitud de la tarifa de la tasa, la contaminación es más alta que la meta, por lo tanto, la tarifa de la tasa no afecta su comportamiento de reducción.

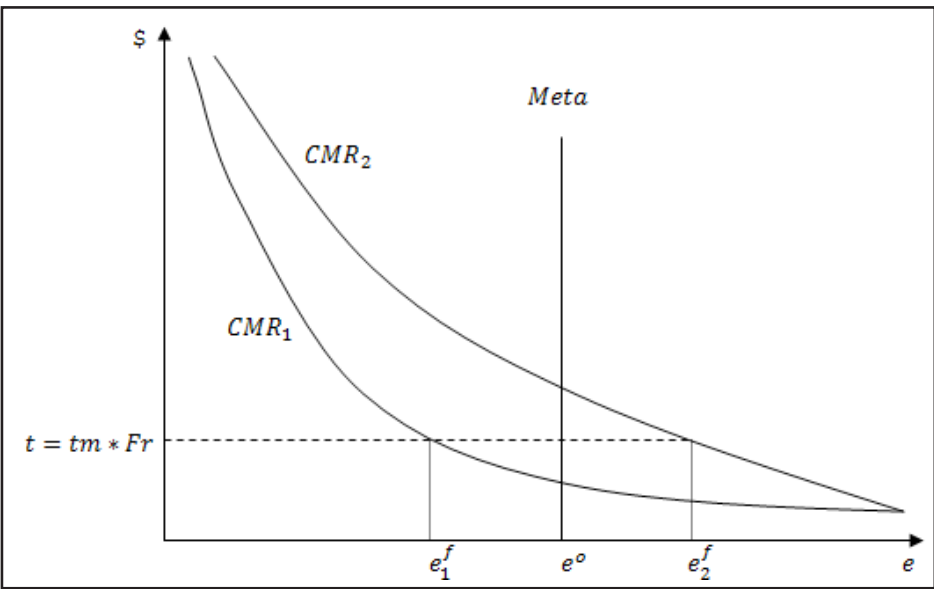

Figura 1. Efecto de la tarifa de la tasa retributiva sobre la contaminación

Lo que se quiere resaltar con el ejemplo de la Figura 1 es que el efecto de la tarifa de la tasa retributiva es heterogéneo. En niveles bajos de la tarifa de la tasa retributiva, ese precio a la contaminación no tendrá efectos de reducción en firmas con costos marginales de reducción altos, pero sí modificara el comportamiento de reducción de firmas con bajo costo de abatimiento.

De allí también surge una explicación del porqué una proporción significati- va de firmas no se preocupa por conocer la tarifa de la tasa. El problema se presenta cuando las metas de reducción de la contaminación no son muy ambiciosas y la tarifa de la tasa es la tarifa mínima, lo cual se da con mucha frecuencia (Rudas, 2008). Empresas con altos costos marginales de reducción se limitan a cumplir con su meta individual de contaminación, porque la reducción adicional es demasiado costosa, por eso prefieren pagar la tasa 
a una tarifa mínima. No es necesario para estas firmas conocer el precio exacto a la contaminación, solo son consientes de que la tarifa es tan baja que su minimización de costos requiere cumplir con la meta de contaminación individual para evitar la sanción que implica la reliquidación del exceso de contaminación a una tarifa más alta, es decir, con un factor regional superior a la unidad.

Evolución de la normatividad sobre las tasas retributivas

Las tasas retributivas tienen como origen el artículo 18 del Decreto 2811 de 1974 (Código de Recursos Naturales Renovables).

Artículo 18. La utilización directa o indirecta de la atmósfera, de los ríos, arroyos, lagos y aguas subterráneas, y de la tierra y el suelo, para introducir desechos o desperdicios agrícolas, mineros o industriales, aguas negras o servidas de cualquier origen, humos, vaporosas y sustancias nocivas que sean resultado de actividades lucrativas, podrá sujetarse al pago de tasas retributivas del servicio de eliminación o control de las consecuencias de las actividades nocivas expresadas.

El artículo no es claro en cuanto a cuál es realmente el objetivo principal de las tasas retributivas ¿el control de la contaminación? o ¿establecer un pago para la eliminación de sus consecuencias? Parece que su intención era que el agente contaminador respondiera por el daño causado.

Diez años más tarde se expide el Decreto 1594 de 1984, en un esfuerzo por reglamentar el artículo 18 del Decreto 2811. El instrumento definido en el decreto reglamentario no persigue la eficiencia económica ni establece metas de contaminación que se puedan asociar con objetivos ambientales, solo se define como un cobro a manera de remuneración al Estado por la prestación de un servicio.

El artículo 18 del Decreto Ley 2811 de 1974 fue derogado por la Ley 99 de 1993, que en el artículo 42 redefinió las tasas retributivas como un modelo de optimalidad paretiana al precisar los factores para el cálculo de la tarifa de la tasa: los costos sociales y ambientales del daño y los costos de recuperación del recurso afectado. Desde el enfoque de la economía ambiental, esta versión de las tasas retributivas puede ser catalogada como un impuesto pigouviano, porque del equilibrio entre ambos conjuntos de costos se puede determinar el nivel óptimo de la contaminación y definir la tarifa de la tasa como el impuesto pigouviano que permita alcanzar dicho óptimo.

Posteriormente se expidió el Decreto 901 de 1997, que reglamentó el cobro de las tasas retributivas con el único 
propósito de incentivar a los usuarios de los cuerpos de agua como receptores de vertimientos, a atenuar sus descargas contaminantes. El Decreto 901 se apartó del modelo de optimalidad paretiana y lo cambió por un instrumento de eficiencia sin optimalidad que, en esencia, sigue las recomendaciones de Baumol y Oates (1971): fijar una meta de reducción de la carga contaminante para el cuerpo de agua y una tasa no pigouviana como precio a la contaminación, que se ajusta paulatinamente hasta alcanzar la meta de reducción establecida.

La meta de contaminación debe ser definida por la autoridad ambiental responsable del cuerpo de agua, para un quinquenio de aplicación de la tasa, y la tarifa de la tasa es el producto de multiplicar la tarifa mínima de la tasa por un factor regional con valor inicial de uno. Ante el cobro del precio uniforme, las empresas decidirían cuánto contaminar, igualando la tarifa de la tasa al costo marginal de reducción de la contaminación, con lo cual se obtiene la equimarginalidad de costos de reducción de la contaminación y se reduce la contaminación al mínimo costo. Si la suma de la contaminación era superior a la meta de contaminación, el factor regional aumentaba en 0,5 unidades cada semestre, incrementando de esa forma el precio a la contaminación y, por ende, la reducción de la contaminación, hasta alcanzar la meta.
Continuando con el registro histórico, el Decreto 901 fue derogado por el Decreto 3100 de 2003. La reglamentación requería que la autoridad ambiental definiera una meta global de reducción de carga contaminante total, y metas individuales y sectoriales para entidades prestadoras de servicio de alcantarillado y otros usuarios cuya carga vertida fuera mayor al $20 \%$ del total de carga que recibía el cuerpo de agua. El factor regional tenía un valor inicial de uno y se ajustaba anualmente a los usuarios que no cumplían con la meta de reducción. Con este nuevo diseño de la tasa, a los contaminadores se les cobran diferentes precios, violando así el principio de equimarginalidad, condición básica para la reducción de costo efectiva de la contaminación. El Decreto 3100 es modificado parcialmente por el Decreto 3440 de 2004. La reforma reglamentó aspectos relacionados con la gestión del cobro de las tasas retributivas por parte de la autoridad ambiental, y la presentación de informes para evaluar su efectividad.

Finalmente, el Decreto 2667 del 2012 (que en su artículo 28 derogó el Decreto 3100 de 2003) contiene la reglamentación vigente de las tasas retributivas. Entre los cambios destacables, el decreto incorporó una sanción para las empresas que no cumplan con la meta individual o grupal de reducción (artículo 17), que consiste en la reliquidación del cobro de la tasa retribu- 
tiva, considerando la diferencia entre el factor regional al final del año, con el factor regional del periodo causado.

Las metas individuales y la sanción convierten el sistema en un híbrido entre un instrumento económico y uno de comando y control, porque se establecen límites de vertimientos (meta) que si no son respetados por las firmas, estas son objeto de una multa que consiste en la reliquidación de la tasa.

\section{El problema de la evaluación del impacto de las tasas retributivas}

Para responder la pregunta: ¿son las tasas retributivas un instrumento eficaz para el control de la contaminación de los cuerpos de agua en Colombia? es necesario estimar el impacto de las tasas retributivas en la moderación de la contaminación de los usuarios con vertimientos. Para este propósito, la aproximación más común es la implementada en estudios de Galarza (2009) y Guerra (2014), en los cuales se especifica un modelo econométrico cuya variable dependiente es la concentración de carga contaminante en los vertimientos de las firmas $\{e\}$ y la variable explicativa de interés es la tarifa de la tasa retributiva $\{t\}$.

$$
e_{i}=\beta_{0}+\gamma * t_{i}+\text { efecto de otros factores }+\varepsilon_{i}
$$

El parámetro $\gamma$ es el efecto marginal de la tarifa de la tasa sobre la conta- minación. La teoría económica sugiere que con el precio a la contaminación, la concentración de las sustancias contaminantes en los vertimientos debe caer, lo que condiciona el planteamiento de las hipótesis nula y alterna:

$$
\begin{aligned}
& H_{o}: \gamma=0 \\
& H_{a}: \gamma>0
\end{aligned}
$$

Bajo la hipótesis nula, las tasas retributivas son inocuas para el control de los vertimientos. Si se rechaza la hipótesis nula, se concluye que el instrumento de precios afecta el comportamiento de los agentes contaminadores incentivándolos a tomar medidas para el control de los vertimientos.

De la regresión de la variable dependiente índice de contaminación $e$ sobre la tarifa de la tasa retributiva y otros factores se obtiene el estimador del impacto de la tarifa ¿Cómo se interpreta $\hat{\gamma}$ ? Dado que, como se advierte en el apartado anterior, el efecto de la tarifa de la tasa sobre la contaminación es heterogéneo, podría pensarse que dicho estimador es el efecto promedio de la tarifa de la tasa retributiva sobre la contaminación, pero esta interpretación no es correcta. Para explicar esta declaración se reformula el modelo [2], considerando la heterogeneidad del efecto de la tarifa de la tasa sobre la carga contaminante presente en los vertimientos. Posteriormente se evalúa el cumplimiento del supuesto de exogeneidad de la tarifa de la tasa en 
la ecuación [3]. Se omite el efecto de otros factores sobre la carga contaminante por simplicidad.

$$
\begin{gathered}
e_{i}=\beta_{0}+\gamma_{i} t_{i}+u_{i} \\
e_{i}=\beta_{0}+\gamma_{i} t_{i}+\gamma t_{i}+u_{i}-\gamma t_{i} \\
e_{i}=\beta_{0}+\gamma t_{i}+u_{i}+\gamma_{i} t_{i}-\gamma t_{i} \\
e_{i}=\beta_{0}+\gamma t_{i}+u_{i}+t_{i}\left(\gamma_{i}-\gamma\right) \\
e_{i}=\beta_{0}+\gamma t_{i}+\varepsilon_{i} \\
\varepsilon_{i}=u_{i}+t_{i}\left(\gamma_{i}-\gamma\right) \\
E\left(\varepsilon_{i} \mid t_{i}\right)=E\left(u_{i} \mid t_{i}\right)+E\left(t_{i}\left(\gamma_{i}-\gamma\right) \mid t_{i}\right)
\end{gathered}
$$

Por el teorema condicional de la ley de expectativas iteradas ${ }^{2}$ :

$$
\left.E\left(\varepsilon_{i} \mid t_{i}\right)=E\left(u_{i} \mid t_{i}\right)+t_{i} E\left(\gamma_{i}-\gamma\right) \mid t_{i}\right)
$$

Si (2b) no tiene errores de especificación, como por ejemplo variables omitidas correlacionadas con la tarifa de la tasa, se tiene que $E\left(u_{i} \mid t_{i}\right)=0$. Sin embargo, $E\left(\gamma_{i}-\gamma \mid t_{i}\right) \neq 0$, porque como se explicó en el apartado anterior $\gamma_{i}$ y $t_{i}$ no son independientes, ya que el efecto individual depende de la tarifa de la tasa retributiva. Se tiene por tanto que $E\left(\varepsilon_{i} \mid t_{i}\right) \neq 0$, y dada la endogeneidad de la tarifa de la tasa en la ecuación [2], el estimador $\hat{\gamma}$ es sesgado e inconsistente, y no es el efecto promedio de la tarifa de la tasa retributiva sobre la concentración de la contaminación.

\section{Literatura previa sobre la eva- luación de las tasas retributivas}

El Ministerio del Medio Ambiente (2002) presentó la evaluación del primer quinquenio de aplicación de las tasas retributivas en Colombia, que incluye los resultados de una primera estimación del efecto de las tasas retributivas sobre las acciones de reducción de los agentes con vertimientos. La información para la estimación econométrica fue de tipo datos panel, sobre las firmas con vertimientos, para los cinco años de aplicación de la tasa retributiva (1997-2002).

Se estimó un modelo logit para probabilidad de reducción de la concentración de la contaminación ante el cobro de las tasas retributivas. La conclusión general del estudio es que los vertimientos reaccionan de manera significativa al cobro de la tasa retributiva. Sin embargo, la reacción no es inmediata, se determinó que la concentración de las sustancias contaminantes en los vertimientos comenzaba a reducirse después de dos períodos de rezago $^{3}$. Por tratarse de un modelo de respuesta binaria, el coeficiente de la variable de precio no midió el impacto de la tarifa sobre la concentración de las sustancias contaminantes en los

\footnotetext{
${ }^{2} \mathrm{E}[g(x) y \mid x]=g(x) E(y \mid x)$.

${ }^{3}$ Periodo comprendido entre el cobro de la tasa y la reacción del agente contaminador, que lo lleva a escoger un nivel de contaminación óptimo que minimice sus costos.
} 
vertimientos, lo que se determinó es que cobrar las tarifas sí tenía un efecto positivo sobre la probabilidad de reducción de concentración de la contaminación en los vertimientos de las empresas.

Rudas (2005) estimó una función de costos de reducción de plantas de tratamiento de aguas residuales, para una muestra de firmas del sector manufacturero en Bogotá. A partir de la derivada de la función de costos de reducción se obtienen los costos marginales. El autor asume que si se cobran tasas por contaminar, cada firma llevará sus vertimientos de aguas servidas hasta un punto en donde el costo marginal por reducirlos sea igual a la tarifa de la tasa. Así se obtiene el impacto de la tasa retributiva en la reducción de la contaminación, comparando la situación inicial o línea base con la contaminación eficiente después de aplicada la tasa. Posteriormente se simulan escenarios para una muestra ampliada de firmas, frente a distintos niveles de tarifas de las tasas. El resultado que se obtiene es que si se cobra la tarifa mínima que fija la norma vigente en Colombia, el impacto sería nulo; pero con la tarifa máxima permitida, se obtendrían reducciones netas de alrededor del $50 \%$ de los vertimientos actuales.

Para la estimación de la función de costos de reducción se utilizó informa- ción disponible para el período 19952001 de firmas manufactureras que operaban en la ciudad de Bogotá y que disponían de planta de tratamiento de aguas residuales, con información técnica sobre la magnitud y efectividad de estas plantas reportada por los monitoreos directos efectuados por el DAMA (Departamento Administrativo del Medio Ambiente) sobre los efluentes de las firmas y con datos sobre las inversiones y los costos de operación del tratamiento de aguas residuales de la Encuesta Anual Manufacturera del Departamento Administrativo Nacional de Estadísticas (DANE).

El problema con la evaluación ex ante del impacto de la tasa desarrollada en Rudas (2005) es que asume racionalidad perfecta de los agentes contaminadores. Se necesita que estos conozcan la tarifa de la tasa, así como su función de costo de reducción de la contaminación y que tengan la habilidad para resolver el problema de minimización de costos por contaminar. Así que esta metodología no es la indicada para la evaluación ex post del impacto, porque ya se tiene como insumo el conocimiento de los niveles de contaminación de las firmas después de aplicado el instrumento.

Galarza (2009) analiza la efectividad de las tasas retributivas en Colombia, utilizando información de los usuarios con vertimientos en la jurisdicción de 
las corporaciones autónomas regionales $^{4}$ CDMB (Corporación Autónoma Regional para la Defensa de la Meseta de Bucaramanga), CORNARE (Corporación Autónoma Regional de las Cuencas de los Ríos Negro y Nare) y CVC (Corporación Autónoma Regional del Valle del Cauca). El número de usuarios industriales fue de $186 \mathrm{em}$ presas y se contó con información para los 10 semestres del período 1998 2002. El panel balanceado le permitió estimar modelos en niveles y en primeras diferencias del impacto de índices de precios de las tarifas de DBO y SST sobre índices de carga contaminante de DBO y SST respectivamente. En el modelo en primeras diferencias, no se encontró efecto de las tarifas sobre la reducción de cargas contaminantes (DBO y SST). Sí se encontraron efectos positivos en el modelo en niveles, pero estos resultados no son confiables, porque la estimación por mínimos cuadrados ordinarios con datos agrupados puede ser sesgada e inconsistente debido a los efectos fijos individuales no observados.

Méndez y Hernández (2012) evaluaron el impacto de la tasa retributiva en la toma de acciones para el control de vertimientos por parte de los agentes contaminadores. Para esto aplicaron una encuesta a los funcionarios del departamento de gestión ambiental de 50 empresas con vertimientos en Bogotá, a fin de conocer su percepción sobre las tasas retributivas, y qué acciones han tomado las empresas como respuesta al cobro de la tasa. El $54 \%$ de los encuestados respondió que percibe la tasa solo como una sanción por contaminar y no como un incentivo económico que los conduzca a moderar sus vertimientos en función de la tarifa de la tasa. En cuanto al valor aproximado de la tarifa de la tasa retributiva para los parámetros de contaminación BBO y SST, el $94 \%$ de los encuestados manifestó no conocerlo.

Con los datos de la encuesta se estimó un modelo probit para establecer los factores por los cuales las empresas tomaban acciones para el control de sus vertimientos. Se concluyó que las empresas que autodeclaran sus vertimientos $\mathrm{y}$, al mismo tiempo, perciben las tasas retributivas como una sanción y no como un impuesto a la contaminación, son las que tienen mayor probabilidad de tomar acciones para el control de sus vertimientos.

Guerra (2014) estimó un modelo econométrico para evaluar la efectividad de las tasas retributivas utilizando datos panel, con información sobre la concentración de DBO y SST en las aguas residuales municipales como variable explicada. La estimación se hizo por mínimos cuadrados ordina-

\footnotetext{
${ }^{4}$ Autoridades ambientales.
} 
rios (MCO) aplicados a los datos agrupados. Se encontró que la tarifa de la tasa retributiva no tiene efectos sobre la reducción de la concentración de las sustancias contaminantes. Tampoco tiene ningún impacto la implementación del plan de manejo y saneamiento de vertimientos. El índice de recaudo, definido como la relación entre el monto recaudado y facturado, sí tiene de efectos positivos y estadísticamente significativos en la reducción de las concentraciones de DBO y SST en los vertimientos.

Una explicación para estos resultados es que las empresas de servicios públicos no tienen incentivos para reducir la concentración de la contaminación construyendo o mejorando sus sistemas de tratamiento de aguas residuales municipales, porque el monto por el pago de la tarifa puede ser transferido a los usuarios (Méndez, 2010). Por otra parte, la estimación por MCO también deja dudas sobre los resultados de la estimación del impacto de las tarifas, al no tener en cuenta la heterogeneidad individual no observada.

\section{METODOLOGÍA}

La estimación del impacto de las tasas retributivas sobre los vertimientos de los agentes contaminadores, desarrollada en este artículo, sigue dos aproximaciones diferentes. La primera asume que el impacto del precio a la contaminación es homogéneo, o que aún siendo heterogéneo el estimador arroja el efecto promedio de las tarifas de la tasa sobre la concentración de sustancias contaminantes. El segundo incorpora la heterogeneidad directamente al modelo, al asumir que cuando las empresas no conocen la tarifa de la tasa, el precio a la contaminación no tiene efecto en las decisiones de reducción de la contaminación de estas firmas. Las estrategias utilizan información de diferentes regiones, porque la información del Ecodirectorio de la Secretaría Distrital de Ambiente de Bogotá facilitó la realización de la encuesta para la estrategia 2, pero en este conjunto de datos no existía la suficiente variabilidad de la tarifa de la tasa para implementar la estrategia de estimación 1, por eso se acudió a la información de CORNARE.

\section{Estrategia de estimación 1}

En esta primera aproximación al problema se estima el efecto de las tarifas de la tasa sobre la concentración de las sustancias contaminantes DBO y SST, suponiendo que las tarifas de la tasa retributiva son información conocida por todos los usuarios con vertimientos, y que estos toman sus decisiones de reducción de la concentración de la contaminación en función de sus precios. Esto es compatible con las estimaciones de antecedente ya reseñadas, porque la información con la que se estiman los modelos econométricos para la evaluación de impacto tiene características similares. 
Los datos para la estimación del efecto de las tarifas provienen de CORNARE (Corporación Autónoma Regional de las Cuencas de los Ríos Negro y Nare). Esta autoridad ambiental es líder en el proceso de implementación de la tasa, y tiene un sistema de información ambiental que facilita la concertación de metas, facturación, monitoreo y no restringe el acceso al público a la consulta de información. De su página web se descargó la información de los usuarios con vertimientos, desde el año 2010 al 2014. Ellos son clasificados según el código del sector económico al que pertenecen, y la cuenca y tramo en la que realizan los vertimientos. Se dispone de información como el tipo de meta de reducción que le impone la autoridad ambiental (global o individual), el factor regional (para DBO y SST), el caudal vertido (en m3/año), las cargas actuales de DBO y SST (en kg/año), la meta al final del quinquenio, la facturación y el recaudo total. El número de usuarios con vertimientos es superior a 200 cada año.

La ventaja de utilizar la información de CORNARE (Corporación Autónoma Regional de las Cuencas de los Ríos Negro y Nare) es que es una autoridad ambiental que implementó las tasas retributivas desde el primer quinquenio de aplicación del instrumento (1997-2002), que desarrolla programas de socialización del instrumento y que no tiene restricciones de acceso a la información, así que las empresas con vertimientos en la jurisdicción de CORNARE están mucho mejor informadas que el promedio nacional.

La especificación del modelo econométrico de la primera alternativa es:

$$
\begin{gathered}
\text { Concentración }_{i, t, j}=\beta_{0, j}+\alpha_{1, j} * F R_{i, t, j}+\beta_{l, j} \\
p_{-} r f_{i, t}+a_{i, j}+\varepsilon_{i, t, j}
\end{gathered}
$$

Donde:

Concentración $_{i, t, j}$ : Concentración de la carga contaminante de la sustancia $\mathrm{j}$, del usuario $\mathrm{i}$, en el periodo $\mathrm{t}$ ( $\mathrm{en} \mathrm{kg}$ / $\mathrm{m} 3$ ).

$F R_{i, t, j}:$ Factor regional para la sustancia j, del usuario i, en el periodo t. Esta variable representa el nivel real de la tarifa de la tasa.

$p_{-} r f_{i, t}:$ Es la relación entre el recaudo total y la facturación.

Se espera que $\alpha_{1}<0$, porque a mayor valor de la tarifa de la tasa, los usuarios tienen mayor incentivo para reducir la contaminación. $\beta_{l}<0$ porque entre más rápido se les cobre a las firmas, más incentivadas estarán en reducir la contaminación.

\section{Estrategia de estimación 2}

Para tener en cuenta la heterogeneidad del efecto de las tarifas de la tasa sobre la reducción de las sustancias contaminantes, en esta segunda estrategia se hizo una encuesta ${ }^{5}$ en la que se

\footnotetext{
${ }^{5}$ Encuesta financia a por el proyecto "Efectividad de las tasas retributivas por contaminación hídrica: el caso de la ciudad de Bogotá. D. C.”, de la Fundación Universitaria Los Libertadores de Colombia.
} 
pregunta a los funcionarios del departamento de gestión ambiental de 315 empresas colombianas con vertimientos, si conocen o no el precio marginal de la tasa retributiva por DBO y SST, y se les pide que respondan cuál es su valor exacto.

La idea es que si en la firma no conocen la tarifa de la tasa, entonces se le asigna un valor de cero a la variable en el modelo de regresión, porque es claro que la empresa no toma sus decisiones de reducción con base en el precio a la contaminación.
La encuesta se realizó telefónicamente entre los años 2014 y 2015, y la lista de empresas fue tomada del Ecodirectorio de la Secretaría Distrital de Ambiente de Bogotá. Se trató de incluir empresas de distintas ramas de la actividad económica, pero haciendo énfasis en industrias cuyo proceso productivo incorporara visiblemente la utilización de agua. Después de un análisis de afinidad entre actividades, se muestra en la Figura 2 la distribución de las empresas encuestadas.

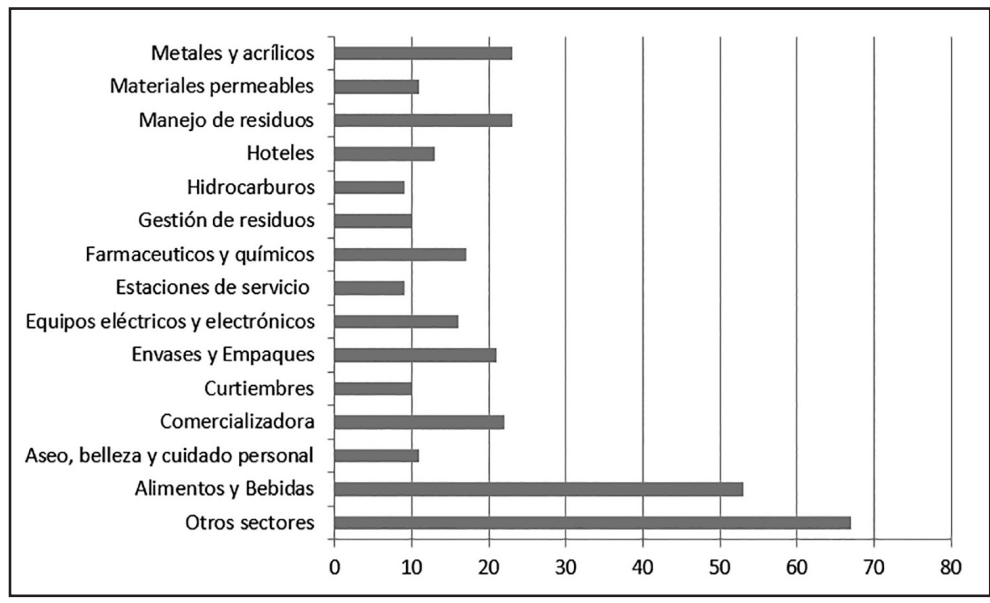

Figura 2. Distribución de la actividad económica de las empresas.

Fuente: elaboración propia con base en datos de encuesta.

El segundo modelo econométrico estima el impacto de las tarifas de la tasa retributiva sobre la reducción de la carga contaminante en las plantas de tratamiento de aguas residuales de las firmas. La especificación del modelo es:

$$
\begin{gathered}
r C C_{i, j}=\beta_{0, j}+\beta_{1, j} Q_{i}+\sum_{k=1}^{K=1} \delta_{k, j} * \text { sector }_{k}+\alpha_{1, j} * t_{j}+\sum_{k=1}^{K=1} \lambda_{k j} * t_{j} * \text { sectork }+ \\
\beta_{1, j} * \text { sancion }_{i}+\beta_{2, j}{ }^{*} \text { responsabilidad }{ }_{i}+\varepsilon_{i, j}
\end{gathered}
$$


Donde:

$i$ : Es el subíndice de firma.

$j$ : Es el subíndice para la sustancia contaminante DBO o SST.

$r C C_{i, j}:$ Es la razón entre las cargas contaminantes de salida $C C s$ y entrada $C C e$ a la PTAR de la empresa contaminadora $i,(\mathrm{CCs} / \mathrm{CCe})$ para la sustancia contaminante $j$. Esta variable corresponde la remoción de la contaminación de la sustancia $j$.

$Q_{i}$ : Caudal de agua tratada por la firma $i$.

sector: conjunto de variables binarias que representan a qué sector económico pertenece cada firma contaminadora.

$t_{j}$ : tarifa de la tasa retributiva para la sustancia contaminante $j$. Variable cuantitativa medida en $\$$. La variable toma el valor reportado en la encuesta por la empresa; si la empresa no conoce el monto de las tarifas de la tasa retributiva para las sustancias contaminantes, su valor es de cero, porque en ese caso, en la práctica, la tarifa no es un incentivo para el esfuerzo de reducción de las firmas. Por otra parte, solo a las firmas que realizan autodeclaración de vertimientos se les cobra en función de sus vertimientos, es decir, son las que tienen un incentivo para reducir la contaminación ante el cobro de las tasas retributivas. Por tanto, solo las firman que hacen autodeclaración y reportan la tarifa de la tasa para cada sustancia contaminante, son las que tienen valor no nulo en esta variable.

Sanción: variable que toma el valor de uno, si la firma percibe la tasa retributiva como una sanción.

Responsabilidad: variable que toma el valor de uno, si la firma respondió que la responsabilidad ambiental es su principal motivo para el control de los vertimientos.

Se espera que $\alpha_{1}<0$, porque a mayor valor de la tarifa de la tasa reportada por la encuesta, los usuarios tienen mayor incentivo para reducir la contaminación

\section{RESULTADOS}

\section{Primera estimación}

Estadisticas descriptivas de las empresas con vertimientos en el área de jurisdicción de CORNARE

La Tabla 1 presenta estadísticas descriptivas de los factores regionales DBO y SST aplicados por CORNARE (Corporación Autónoma Regional de las Cuencas de los Ríos Negro y Nare) a las empresas con vertimientos en su jurisdicción, durante los años 20112014. Se advierte que, para todos los años, a la mayoría de las empresas se les cobra como tarifa de la tasa la tarifa mínima, es decir, que el factor regional aplicado es de 1 . Se puede observar 
también que los factores regionales cayeron en el año 2012 en comparación con el año 2011, para después incrementarse en los años 2013 y 2014. Se puede ver, además, que históricamente los mayores incrementos en el factor regional y por tanto en la tarifa real en la tasa se dan en los sólidos suspendidos totales.

La Tabla 2 muestra las estadísticas descriptivas de la concentración de las sustancias contaminantes DBO y SST en los e luentes de las empresas en el área de jurisdicción de CORNARE. Se observa que la tendencia en la concentración de las sustancias contaminantes es decreciente, lo cual teóricamente es compatible con el incremento del precio a la contaminación para los sólidos suspendidos totales, pero para la demanda bioquímica de oxígeno no se puede asegurar que exista correlación lineal entre el precio promedio de la contaminación por DBO y concentración de la contaminación promedio por DBO.

Tabla 1. Estadísticas descriptivas de los factores regionales DBO y SST aplicados por CORNARE

\begin{tabular}{|c|c|c|c|c|}
\hline \multicolumn{5}{|c|}{$\begin{array}{l}\text { Porcentaje de empresas con tarifa de la tasa } \\
\text { igual a la tarifa mínima }\end{array}$} \\
\hline & \multicolumn{2}{|c|}{ FR-DBO } & \multicolumn{2}{|c|}{ FR-SST } \\
\hline 2011 & \multicolumn{2}{|c|}{$60.4 \%$} & \multicolumn{2}{|c|}{$76.1 \%$} \\
\hline 2012 & \multicolumn{2}{|c|}{$85.2 \%$} & \multicolumn{2}{|c|}{$84.7 \%$} \\
\hline 2013 & \multicolumn{2}{|c|}{$79.62 \%$} & \multicolumn{2}{|c|}{$59.2 \%$} \\
\hline 2014 & \multicolumn{2}{|c|}{$76.40 \%$} & \multicolumn{2}{|c|}{$53.4 \%$} \\
\hline \multicolumn{5}{|c|}{ Media y desviación estándar del factor regional } \\
\hline & \multicolumn{2}{|c|}{ FR-DBO } & \multicolumn{2}{|c|}{ FR-SST } \\
\hline & Promedio & D. estándar & Promedio & D. estándar \\
\hline 2011 & 2.25 & 0.13 & 1.83 & 0.12 \\
\hline 2012 & 1.56 & 0.11 & 1.62 & 0.12 \\
\hline 2013 & 1.73 & 0.12 & 2.21 & 0.13 \\
\hline 2014 & 1.80 & 0.12 & 2.81 & 0.16 \\
\hline
\end{tabular}


Tabla 2. Estadísticas descriptivas de la concentración de las sustancias contaminantes DBO y SST

\begin{tabular}{|c|c|c|c|c|}
\hline \multicolumn{4}{|c|}{ Media y desviación estándar de la concentración de la contaminación } \\
(en mg/l) \\
\hline \multirow{2}{*}{} & \multicolumn{2}{|c|}{ FR-DBO } & \multicolumn{2}{c|}{ FR-SST } \\
\cline { 2 - 5 } & Promedio & D. estándar & Promedio & D. estándar \\
\hline 2011 & 210 & 40 & 130 & 10 \\
\hline 2012 & 170 & 20 & 120 & 10 \\
\hline 2013 & 160 & 20 & 120 & 10 \\
\hline 2014 & 160 & 20 & 110 & 10 \\
\hline
\end{tabular}

\section{Estimación del modelo 1}

Los resultados de la estimación de los efectos de las tarifas de la tasa retributiva sobre la concentración de sustancias contaminantes por efectos fijos y aleatorios se presentan en la Tabla 3 . La misma tabla muestra los resultados del test de Hausman para las ecuaciones de DBO y SST. Sobre la ecuación de DBO, el test de Hausman no encuentra diferencias estadísticamente significativas entre efectos fijos y alea-torios ( $p$ valor $=0.37>\alpha=$ $0.05)$. En cuanto a la ecuación de SST, el test de Hausman haya diferencias estadística-mente significativas entre efectos fijos y aleatorios, lo que favorece la estimación por efectos fijos

$$
\text { ( } p \text { valor }=0.009<\alpha=0.05) \text {. }
$$

En la ecuación de carga contaminante de la demanda bioquímica de oxígeno DBO, el coeficiente del factor regional de la tasa por DBO (valor real de la tarifa) tiene el signo esperado, pero el efecto de la tarifa no es significativo en las estimaciones por efectos fijos y aleatorios. Lo que evidencian las estimaciones es que la reducción de la concentración de carga contaminante de DBO depende de la facturación y cobro de la tasa retributiva, porque la relación entre el recaudo total y facturación a la empresa sí resulta significativa hasta el $1 \%$.

En la estimación por efectos fijos de la ecuación de carga contaminante de sólidos suspendidos totales (SST) elegida mediante el test de Hausman, se obtuvo que tanto el coeficiente del factor regional de la tasa por SST, como la facturación y cobro de la tasa retri- 
butiva tienen el signo esperado y las variables son significativas al $5 \%$ y $1 \%$ respectivamente.

Lo que se concluye de este primer ejercicio econométrico es que la tarifa real de la tasa retributiva no tiene efecto sobre carga contaminante de materia orgánica (DBO). Por otra parte, aumentar el factor regional de SST en una unidad, lo que implica el incremento de la tarifa de la tasa retributiva en una cuantía igual a la magnitud de la tarifa mínima para SST, tiene un efecto de reducción sobre la concentración de la sustancia contaminante de 10 según la estimación por efectos fijos. La concentración promedio de SST de las empresas es de 133, así que la reducción promedio sería del $7.5 \%$. También se concluye que la facturación y cobro de la tasa retributiva sí induce en los agentes contaminadores un comportamiento más cooperativo con el medio ambiente.
Los resultados no sorprenden, porque siguen la línea de la explicación teórica del apartado dos. El porcentaje de firmas a las cuales se les cobra una tarifa por encima de la tarifa mínima es superior para los sólidos suspendidos totales, en comparación con la demanda bioquímica de oxígeno, así que más firmas estarían incentivadas para reducir SST que DBO. También la tarifa real promedio de la tasa alcanza valores más altos en sólidos suspendidos totales, así que el nivel promedio del incentivo es más alto para SST. Esto explica por qué el efecto del precio a la contaminación sobre la concentración de sustancia contaminante es significativo en la ecuación de SST.

Por otra parte, a partir de las estadísticas descriptivas no se puede concluir que exista correlación lineal entre la tarifa promedio de DBO y la concentración promedio de DBO en los efluentes.

Tabla 3. Estimación con datos panel. Variable dependiente: concentración de sustancia contaminante

\begin{tabular}{|l|c|c|c|c|}
\hline \multirow{2}{*}{ Regresores } & \multicolumn{2}{|c|}{ Concentración de DBO } & \multicolumn{2}{c|}{ Concentración de SST } \\
\cline { 2 - 5 } & Efectos fijos & $\begin{array}{c}\text { Efectos } \\
\text { aleatorios }\end{array}$ & Efectos fijos & $\begin{array}{c}\text { Efectos } \\
\text { aleatorios }\end{array}$ \\
\hline Factor regional para DBO & -.009 & -.004 & $-.010^{\star *}$ & $-.007^{\star}$ \\
\hline & {$[.007]$} & {$[.006]$} & {$[.005]$} & {$[.004]$} \\
\hline $\begin{array}{l}\text { Recaudo } \\
\text { total/facturación }\end{array}$ & $-.089^{\star * *}$ & $-.081^{* * *}$ & $-.062^{* * *}$ & $-.054^{* *}$ \\
\hline & {$[.031]$} & {$[.028]$} & {$[.023]$} & {$[.021]$} \\
\hline
\end{tabular}


El impacto de las tasas retributivas para el control de vertimientos en Colombia

Johanna Mildred Méndez-Sayago - JhonAlexánderMéndez-Sayago - Hugo Alfonso Hernández Escolar

\begin{tabular}{|l|c|c|c|c|}
\hline Intercepto & .268 & .257 & .195 & .177 \\
\hline & {$[.026]$} & {$[.036]$} & {$[.020]$} & {$[.024]$} \\
\hline Número de observaciones: & 742 & 742 & 742 & 742 \\
\hline Número de grupos: & 194 & 194 & 194 & 194 \\
\hline R-cuadrado: & 0.0227 & 0.0222 & 0.0227 & 0.0432 \\
\hline Test de Hausman: & p_valor $=$ & 0.3782 & p_valor $=$ & 0.0099 \\
\hline
\end{tabular}

Errores estándar robustos entre paréntesis.

$* * *$ p-valor $<0.01, * *$ p-valor $<0.05, *$ p-valor $<0.10$

\section{Estrategia de estimación 2}

\section{Resumen de los resultados de la en- cuesta}

Ante la pregunta ¿cuál es el valor aproximado de la tarifa de la tasa retributiva para DBO y SST? el $79 \%$ de los encuestados manifestó no conocerla. Solo 66 de las 315 firmas conocen y reportan las tarifas de la tasa retributiva. En todos los casos, el valor reportado coincide con las tarifas mínimas por DBO y SST para el año 2014 o el valor del año 2013. Esto evidencia que realmente las firmas no conocen la tarifa de la tasa, que es el producto de la tarifa mínima por el factor regional.

La encuesta encontró que la mayoría de empresas realiza sus vertimientos a la red de alcantarillado (98\%), y solo el $2 \%$ de las empresas son usuarios directos de los cuerpos de agua como receptores de vertimientos. Esta información es importante, porque los usuarios directos son considerados el sujeto pasivo por la normatividad de las tasas retributivas, es decir, pagan directamente el monto de la tasa a la autoridad ambiental. Cuando las empresas realizan vertimientos a la red de alcantarillado, el sujeto pasivo es la empresa de servicios públicos (ESP), y esta traslada el cobro de la tasa a sus usuarios, según lo dispuesto en la Resolución 287 de 2004 de la Comisión Reguladora de Agua Potable y Saneamiento Básico (CRA). Esto último requiere que la ESP exija a sus usuarios la autodeclaración de sus vertimientos, pues, de lo contrario, el cobro de la tasa será sobre unos niveles de contaminación presuntivos.

El $39 \%$ de las empresas encuestadas no realiza autodeclaración de sus vertimientos de DBO y SST. La normatividad ambiental obliga a las autoridades ambientales a solicitar autodeclaraciones de contaminación al sujeto pasivo de la tasa. Sin embargo, cuando el sujeto pasivo es la empresa 
de servicios públicos, esta exigencia parece ser relajada en muchos casos, como lo demuestra la encuesta. Bajo esta limitación administrativa, la tasa retributiva no funciona bien como un incentivo económico para la reducción de la contaminación.

La Figura 3 revela cuál es la percepción de los encargados del departamento de gestión ambiental de las empresas sobre el instrumento tasas retributivas. Obsérvese que una gran cantidad de empresas (71 de 315) percibe la tasa retributiva como una sanción por contaminar, y no como lo que se pretende, que sea un incentivo económico (precio a la contaminación), que los lleve a tomar acciones que conduzcan a moderar sus vertimientos en función de la tarifa de la tasa. Un número de 25 de 315 empresas percibe las tasas retributivas como un cobro simbólico, así que sería lógico que estas empresas no tomen acciones para modificar su contaminación.

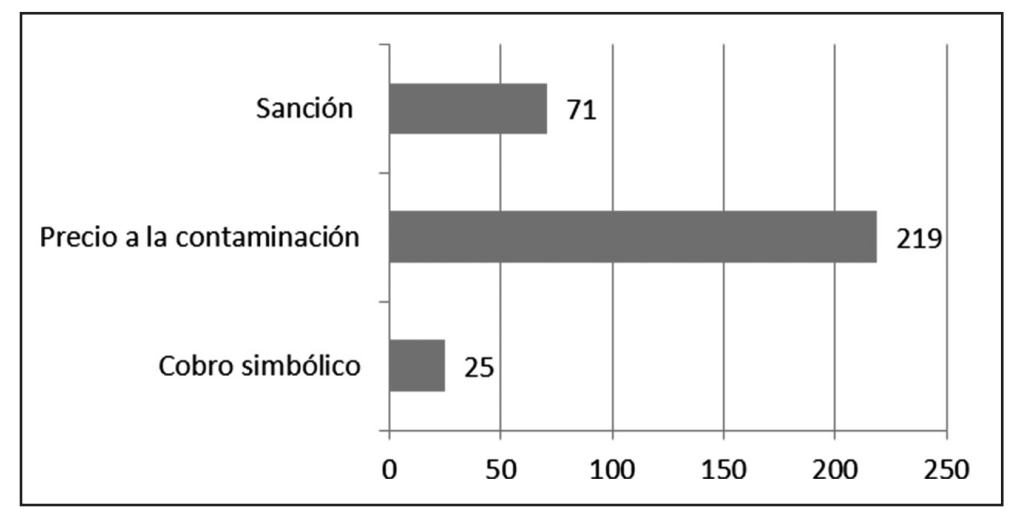

Figura 3. Percepción sobre las tasas retributivas

Como resultado de la aplicación de las tasas retributivas, el $26 \%$ de las empresas encuestadas manifiesta que han cambiado insumos para moderar su contaminación, el $22 \%$ ha modificado sus procesos productivos, y el $33 \%$ realizó inversiones para mejorar sus sistemas de tratamiento. Se reconoció por parte de los encuestados que en los casos en que las repercusiones ambientales motivaron un cambio es- tratégico, ello tuvo origen principalmente en la responsabilidad ambiental $(81 \%)$ y no por el cobro de la tasa retributiva (19\%).

\section{Estimación del modelo 2}

La reducción de la concentración de las sustancias contaminantes se realiza en la misma planta de tratamiento para cada firma, así que existe un solo 
proceso de descontaminación conjunto, y por tanto, es posible que existan factores inobservables o no medidos en la encuesta, que afecten al mismo tiempo la reducción de DBO y SST. Estos factores no observados, al no ser incluidos en las regresiones especificadas por la ecuación [4], generan correlación contemporánea entre el error de la ecuación de reducción de DBO y el error de la ecuación de SST. La estimación eficiente de los parámetros de la ecuación [5] requiere, por tanto, una técnica de estimación que incorpore dicha correlación contemporánea. El estimador de mínimos cuadrados generalizados factibles que aprovecha esta información disponible es el sistema de ecuaciones aparentemente no relacionadas SUR de Zellner (1962).

Sin embargo, antes de realizar la estimación SUR es importante establecer la existencia de correlación "contemporánea" entre los errores de las ecuaciones de reducción de DBO y $\mathrm{SST}^{6}$. En esta aplicación solo hay un corte transversal, así que no existe correlación contemporánea, lo que se evalúa es si los errores de las dos ecuaciones de reducción de la contaminación (DBO y SST) están correlacionados.

El valor del estadístico de prueba es $\lambda=109.07$. Como este valor es superior a $\chi_{(1,0.05)}=3.84$ se rechaza la hipótesis nula y se concluye que sí existe correlación entre los errores de las dos ecuaciones y se debe utilizar el estimador SUR.

Los resultados de la estimación de las ecuaciones de reducción de las concentraciones de las sustancias contaminantes DBO y SST en la planta de tratamiento mediante la técnica SUR se presentan en la Tabla 4. Los efectos son los esperados (positivos) y además son significativos. Un incremento de $\$ 1$ en las tarifas de DBO y SST tiene un efecto de reducción de la concentración de DBO del 0,089\% y del 0,17\% para SST. La responsabilidad ambiental también tiene efecto positivo y significativo en las reducciones de DBO y SST.

\footnotetext{
${ }^{6}$ La hipótesis nula de la prueba de correlación contemporánea asume su no existencia: $H_{0}: \sigma_{12}=0$ $H_{a}$ :al men os una de las covarianzas no es cero

El estadístico de prueba es $1=T \sum_{i=2}^{M} \sum_{j=1}^{i-1} r_{i j}^{2} \sim \mathrm{c}^{2}{ }_{M(M-1) / 2}$. Donde $r^{2}{ }_{i j}=\mathrm{s}_{i j}{ }_{i j} / \mathrm{s}_{i i} \mathrm{~s}_{j j}$ y $M$ es el número de ecuaciones.
} 
Tabla 4. Estimación SUR. Variable dependiente: reducción de la concentración de las sustancias contaminantes en la plata de tratamiento (en porcentaje)

\begin{tabular}{|l|c|c|}
\hline \multicolumn{1}{|c|}{ Variables } & Reducción de DBO & Reducción de SST \\
\hline \multicolumn{1}{|c|}{ Tarifa de la tasa } & $.00089^{* *}$ & $.00170^{* *}$ \\
& {$[.00031]$} & {$[.00053]$} \\
\hline \multicolumn{1}{|c|}{ Responsabilidad ambiental } & $.08969^{\star \star}$ & $.05485^{\star *}$ \\
& {$[.00328]$} & {$[.02557]$} \\
\hline \multicolumn{1}{|c|}{ Binarias de sector } & & \\
\hline Alimentos y bebidas & .51239 & .66733 \\
Aseo, belleza y cuidado personal & .58927 & .76533 \\
Comercializadora & .55639 & .77962 \\
Curtiembres & .64330 & .77163 \\
Envases y empaques & .55176 & .65589 \\
Equipos eléctricos y electrónicos & .56014 & .72445 \\
Estaciones de servicio & .41690 & .67955 \\
Farmacéuticos y químicos & .48024 & .6561 \\
Gestión de residuos & .63141 & .75663 \\
Hidrocarburos & .40811 & .63556 \\
Hoteles & .46807 & .67661 \\
Manejo de residuos & .57570 & .68386 \\
Materiales permeables & .57169 & .72514 \\
Metales y acrílicos & .59392 & .74266 \\
Otros sectores & .50227 & .68567 \\
\hline Número de observaciones & 296 & 296 \\
R-cuadrado & 0.43 & 0.47 \\
\hline
\end{tabular}

Errores estándar robustos entre paréntesis. ${ }^{* * *} \mathrm{p}$-valor $<0.01, * * \mathrm{p}$-valor $<0.05$

Las tarifas mínimas para el año 2014 fueron de de DBO y de SST. La Tabla 5 muestra que el incremento del $50 \%$ de la tarifa mínima reduciría la concentración de la contaminación de
DBO en $5.29 \%$ y de SST en $4.31 \%$. Un incremento del $100 \%$ de la tarifa mínima reduciría la concentración de DBO en $10.58 \%$ y de SST en $8.63 \%$. Un incremento del $200 \%$ de la tarifa 
mínima reduciría la concentración de la contaminación de DBO en $21.16 \%$ y de SST en $17.25 \%$. Pero esto solo para las empresas que realmente perciben la tasa retributiva como un incentivo económico para el control de la contaminación. Para encontrar el efecto promedio deben multiplicarse estos efectos por el porcentaje de empresas que sí toman sus decisiones de reducción en función del precio a la contaminación.

Tabla 5. Simulación de incrementos del precio de la tasa

\begin{tabular}{|c|c|c|c|c|c|c|c|c|}
\hline & & & \multicolumn{6}{|c|}{ Incremento como porcentaje de la tarifa mínima } \\
\hline \multicolumn{2}{|c|}{$\begin{array}{l}\text { Efecto marginal del } \\
\text { precio de la tasa }\end{array}$} & & \multicolumn{2}{|c|}{$50 \%$ de la TM } & \multicolumn{2}{|c|}{$100 \%$ de la TM } & \multicolumn{2}{|c|}{$200 \%$ de la TM } \\
\hline DBO & SST & & DBO & SST & DBO & SST & DBO & SST \\
\hline .00089278 & .00170204 & $\begin{array}{l}\text { Porcentaje } \\
\text { de reduc- } \\
\text { ción }\end{array}$ & $5,29 \%$ & $4,31 \%$ & $10,58 \%$ & $8,63 \%$ & $21,16 \%$ & $17,25 \%$ \\
\hline
\end{tabular}

\section{CONCLUSIONES}

Este artículo presenta argumentos teóricos y evidencia empírica que demuestra que las estimaciones econométricas tradicionales utilizadas para estimar el impacto del precio de la contaminación sobre la concentración de las sustancias contaminantes, pueden arrojar estimaciones sesgadas e inconsistentes de su efecto debido a la endogeneidad de la tarifa de la tasa en la ecuación de concentración o reducción de carga contaminante. La endogeneidad tiene origen en la heterogeneidad del efecto del precio a la contaminación sobre la contaminación de las firmas. El problema es que los efectos individuales de las tarifas sobre la concentración de la contaminación (nulo o positivo) dependen de la magnitud de las tarifas, así que la relación entre tarifas y contaminación es bidireccional.

La encuesta desarrollada evidencia que no todas las empresas se preocupan por conocer las magnitudes de las tarifas de la tasa retributiva por DBO y SST. Es posible que las empresas que tienen costos marginales de reducción altos y enfrentan tarifas de la tasa pequeñas como la tarifa mínima, se limiten a cumplir con su meta individual de contaminación porque la reducción adicional es demasiado costosa, por eso prefieren pagar la tasa a una tarifa mínima. No es necesario para estas firmas conocer el precio exacto a la contaminación, solo son consientes de que la tarifa es tan baja que su minimización de costos requiere cumplir con la meta de contaminación individual para evitar la sanción que implica la reliquidación del exceso de contami- 
nación a una tarifa más alta, es decir, con un factor regional superior a la unidad. Para estas empresas, el efecto de la tarifa sobre los esfuerzos de contaminación es nulo.

Al realizar la estimación tradicional, asumiendo que las firmas tienen conocimiento exacto del precio a la contaminación y por tanto su efecto es homogéneo, se encontró que solo la tarifa de la tasa de sólidos suspendidos totales (SST) tiene efecto sobre la concentración de la contaminación de la misma sustancia contaminante en los efluentes de las firmas. Esto puede ser atribuido a que la tarifa de la tasa por SST alcanza un valor promedio más alto que la de DBO y existe un mayor porcentaje de firmas con tarifas de SST por encima de la tarifa mínima, en comparación con las tarifas de DBO. Esta es una conclusión que coincide con el supuesto hecho sobre cuáles firmas realizan esfuerzos de reducción de la contaminación ante el cobro de la tarifa de la tasa retributiva.

Cuando se estimó el impacto de la tarifa de la tasa retributiva sobre la reducción de la concentración de las sustancias contaminantes en las plantas de tratamiento, asumiendo que el precio a la contaminación no es la tarifa real sino el conocimiento del precio que tiene el agente contaminador, se encontró evidencia estadística de que el efecto de las tarifas es significativo para DBO y SST. Sin embargo, estos no son los efectos promedio de las tarifas de la tasa sobre la reducción de la concentración de DBO y SST, son los efectos sobre las firmas que perciben las tasas retributivas realmente como un precio a la contaminación.

Con ayuda de los efectos marginales encontrados en el modelo con las características descritas anteriormente, se determinó que lograr reducciones adicionales en la concentración de los contaminantes requiere grandes incrementos en las tarifas de la tasa retributiva. Considerando que las tarifas mínimas para DBO y SST para el año 2014 fueron, en su orden, 118.52 $(\$ / \mathrm{kg})$ y $50,68(\$ / \mathrm{kg})$, un incremento del $50 \%$ de la tarifa mínima reduciría la concentración de la contaminación de DBO en $5.29 \%$ y de SST en $4.31 \%$. Un incremento del $100 \%$ de la tarifa mínima reduciría la concentración de DBO en $10.58 \%$ y de SST en $8.63 \%$. Un incremento del $200 \%$ de la tarifa mínima reduciría la concentración de la contaminación de DBO en $21.16 \%$ y de SST en $17.25 \%$.

Se puede concluir que las tasas retributivas sí logran modificar el comportamiento de las firmas y las inducen a tomar acciones destinadas a moderar sus vertimientos, por diferentes razones. Una es la responsabilidad ambiental, la otra se presenta cuando las empresas realmente perciben que la tasa retributiva es un precio a la contaminación. También resulta importante el proceso de facturación y cobro de la tasa, pues sin el buen desempeño administrativo, el instrumento pierde efectividad. 


\section{REFERENCIAS}

Baumol W. \& Oates, W. (1971). The Use of Standards and Prices for Protection of the Environment. Swedish Journal of Economics, 73, 42-54.

Comisión Reguladora de Agua Potable y Saneamiento Básico. (2004). Resolución 287 de 2004. Bogotá: CRAPSB.

Congreso de la República de Colombia. (1993). Ley 99 de 1993, Por la cual se crea el Ministerio del Medio Ambiente, se reordena el Sector Público encargado de la gestión y conservación del medio ambiente y los recursos naturales renovables, se organiza el Sistema Nacional Ambiental-SINA-y se dictan otras disposiciones. Bogotá: Imprenta Nacional.

Galarza, M. (2009). Análisis de la efectividad de las tasas retributivas en Colombia. Estudio de caso. Trabajo de grado Maestría en Economía, Universidad Javeriana, Bogotá.

Guerra, M. (2014). Impacto del programa de tasas retributivas en Colombia. Monografía presentada para optar por el título de economista. Universidad Piloto de Colombia. Bogotá.

Instituto de Hidrología, Meteorología y Estudios Ambientales -IDEAM-. (2014). Estudio nacional del agua. Bogotá: IDEAM.

Méndez, J. (2010). Un sistema de permisos negociables para el control de la contaminación de empresas con vertimientos a la red de alcantarillado. Revista Sociedad y Economía, 18, 241-265.

Méndez, J. \& Hernández, H. (2012). La racionalidad limitada de los agentes contaminadores y sus efectos sobre la eficiencia económica en el control de vertimientos: el caso de las tasas retributivas. Producción + Limpia, 7(1), $32-47$.

Ministerio de Ambiente, Vivienda y Desarrollo Territorial. (2003). Decreto 3100 de 2003. Tasas retributivas por la utilización directa del agua como receptor de vertimientos puntuales. Bogotá: Imprenta Nacional. 
Apuntes CENES Volumen 36, Número 64

julio - diciembre 2017. Págs. 167-198

Ministerio de Ambiente, Vivienda y Desarrollo Territorial. (2004). Decreto 3440 de 2004, por el cual se modifica el Decreto 3100 de 2003. Bogotá: Imprenta Nacional.

Ministerio de Ambiente y Desarrollo Sostenible. (2012). Decreto 2667 de 2012, por el cual se reglamenta la tasa retributiva por la utilización directa e indirecta del agua como receptor de los vertimientos puntuales, y se toman otras determinaciones. Bogotá: Imprenta Nacional.

Ministerio del Medio Ambiente. (1997). Decreto 901 de 1997, sobre tasas retributivas por la utilización directa del agua como receptor de vertimientos puntuales. Bogotá: Imprenta Nacional.

Ministerio del Medio Ambiente. (2002, julio). Evaluación nacional del programa de tasas retributivas. Resumen ejecutivo. Bogotá: MA.

Oficina de Análisis Económico del Ministerio del Medio Ambiente. (1997). Aguas limpias para Colombia al menor costo. Implementación de las tasas retributivas por contaminación hídrica. Palmas, 18(3), 59-69.

Presidente de la República de Colombia. (1984). Decreto 1594 de 1984, usos del agua y residuos líquidos. Bogotá: Imprenta Nacional.

Presidente de la República de Colombia. (2002). Código de Recursos Naturales Renovables y Protección al Medio Ambiente, Decreto-ley 2811 de 1974. Bogotá: Legis.

Rudas, G. (2005, marzo). Instrumentos económicos y regulación de la contaminación industrial: primera aproximación al caso del río Bogotá (Colombia). En Segundo Congreso Latinoamericano de Economistas Ambientales $y$ de Recursos Naturales (ALEAR), Oaxaca, México.

Rudas, G. (2008). Instrumentos económicos en la politica del agua en Colombia: tasas por el uso del agua y tasas retributivas por vertimientos contaminantes. Bogotá: Sistema Nacional Ambiental -SINA-. 
Velásquez, C. (2002). Financiación de la gestión ambiental en Colombia: el caso de las tasas. Revista de Derecho Universidad del Norte, (18), 151-171.

Villegas, C., Castiblanco, C., Berrouet, L. \& Vidal, L. (2006). El programa de tasas retributivas en Colombia y el fortalecimiento institucional de las Corporaciones Autónomas Regionales. Gestión y Ambiente, 9(1), 7-24.

Zellner, A. (1962). An Efficient Method of Estimating Seemingly Unrelated Regressions and Tests for Aggregation. Journal of the American Statistical Association, 57(298), 348-368. 